\title{
Continuous assessment of left ventricular shape in man
}

\author{
D. G. Gibson and D. J. Brown \\ From the Brompton Hospital, London
}

Frame by frame analysis of left ventriculograms has been performed in Io normal subjects and 40 patients with heart disease. Left ventricular shape index was derived as $4 \pi$ (cavity area) $/(\text { perimeter })^{2}$, which has a maximum value of $I$ when the outline is circular. In normal subjects systole was always associated with progressive reduction in shape index, indicating that the cavity projection had become less circular. This change was smaller in patients with low ejection fraction and also when inferior or anterior hypokinesia was present, even though ejection fraction was normal. During early diastole shape index rose rapidly due to an increase in minor diameter occurring throughout the period of rapid filling. In some cases this preceded any change in long axis, which was due to upward movement of the aortic root as well as outward movement of the apex. These results have functional implications, suggesting in particular that wall movement during filling may be non-uniform and that assumptions about cavity shape used in the derivation of wall properties from estimates of ventricular volume may require modification.

Estimation of ventricular volume by angiocardiography has proved a valuable method of studying cardiac function in man. Injection of radio-opaque contrast medium delineates the left ventricular cavity so that its projection in one or two planes can be recorded with high resolution using currently available cineangiographic techniques. However, such studies cannot be used to give direct information about the performance of the heart muscle itself unless a number of other factors including shape changes, cavity obliteration, and endothelial infolding are considered. The purpose of the present study was to define, in more general terms, changes occurring in the shape of the image of the opacified left ventricular cavity throughout the cardiac cycle by performing frame by frame analysis of cineangiocardiograms. It was not necessary to assume that the cavity could be represented by any specific geometrical figure, such as an ellipsoid, but instead a less arbitrary definition of shape was used. In this way it was possible to detect and quantify spontaneous variations in shape occurring in the cardiac cycle, and so to assess the extent to which they, rather than shortening or distension of the ventricular wall, were responsible for changes in cavity size during filling or ejection. Using this approach, the cavity, defined as the region accessible

Received 4 April 1975. to angiographic dye, can be treated as an isolated system whose volume changes form the basis of the cardiac output. Though defined independently of anatomical considerations the properties of this system must be predictable from any model seeking to give a comprehensive picture of left ventricular function.

\section{Methods}

\section{Definition of shape}

Though shape may be defined mathematically in several ways the method used in the present study concerns itself with the relation between the volume of the ventricular cavity and the behaviour of its boundary, and, in particular, with the ratio of the volume of blood to the surface area of ventricular wall in contact with it. If surface area is fixed the volume of blood is at a maximum when the cavity is spherical and drops to zero if complete cavity obliteration occurs. An index of shape expressing this idea might therefore be defined as the volume enclosed by unit surface area of wall. The present study was based on single plane angiocardiograms, so the corresponding quantity is the relation between cavity area and perimeter. In order to make it dimensionless we have defined shape index as

$$
4 \pi \text { (Area) } /(\text { Perimeter })^{2}
$$

which has a maximum value of $I$, or $100 \%$, when the cavity projection is circular and a minimum of zero when cavity obliteration occurs (Holt and Marjoram, 1973). 
Changes in cavity shape can thus be studied independently of those in area or long axis, and the extent to which they occur during ventricular filling or ejection can be assessed.

\section{Material}

The 50 patients studied fell into four diagnostic groups.

(I) Normal subjects (10). These patients had chest pain but haemodynamics, left ventriculography, and coronary arteriography were all within normal limits.

(2) Non-rheumatic mitral regurgitation (18). These patients all subsequently underwent mitral valve replacement for mitral regurgitation due to ruptured chordae tendineae, though a varying degree of left ventricular disease was also present.

(3) Congestive cardiomyopathy (6). Patients with dilated left ventricular cavities and no evidence of significant valvular heart disease. Coronary arteriograms were normal.

(4) Ischaemic heart disease (I6). Two types of patient were studied - those with localized areas of reduced amplitude of wall movement involving the anterior or inferior surface (ro patients), and those considered for excision of left ventricular aneurysm (6 patients).

All the patients were undergoing cardiac catheterization and left ventriculography for diagnostic purposes.

\section{Catheter techniques}

The patients were all premedicated with amylobarbitone $200 \mathrm{mg}$ by mouth one hour before the start of the procedure. Angiocardiography was performed with the patients in either the anteroposterior or the right anterior oblique position, with injection of Triosil $40 \mathrm{ml}$ into the left ventricle at a flow rate of $25 \mathrm{ml} / \mathrm{s}$. Cine film was exposed at 50 frames/s. In 15 patients biplane cineangiograms were performed in the posteroanterior and lateral projections. A simultaneous electrocardiogram was recorded together with an exposure marker so that the frame speed could be checked. Ectopic and postectopic beats were not studied. Calibration was by means of a grid exposed at mid-chest level.

\section{Digitization of cineangiograms}

Cineangiograms were digitized by projection of successive frames of the beat to be studied, starting with that immediately after the $Q$ wave of the electrocardiogram, on to a DMAC. digitizing table interfaced with an IBM. I800 computer (Gibson and Brown, I975). The perimeter of the left ventricular cavity was outlined with a pencil follower starting from the aortic root. Co-ordinates of approximately 50 points were recorded for each cine frame, together with calibration data and a reference point in the thoracic cage or on the border of the cine frame, and stored on magnetic tape.

\section{Analysis of data}

Information derived from the angiograms was displayed in two ways. A simple display (Fig. I) showed successive cavity outlines superimposed, those from the start of the beat being studied to the one with the smallest area being taken as systolic and the remainder as diastolic. This gave pictorial information about patterns of wall movement in different parts of the cavity. Data were also plotted, after smoothing with a three-point moving average filter, as a series of graphs showing variations in area, estimated volume, perimeter, shape index, and long axis, with time after the start of the $Q$ wave. Cavity area was derived by numerical integration and long axis as the distance from the mid-point of the aortic root to the furthest point on the perimeter. Neither of these two derivations, therefore, make any assumptions about cavity shape. Estimates of cavity volume, which were used only to calculate ejection fraction, were made from area and expressed in normalized form to indicate that they had not been measured directly.

Standard $t$ tests were used to examine differences between means.

\section{Results}

The results are shown in detail in the Table.

\section{Normal subjects}

The mean ejection fraction $( \pm S D)$ in these patients was $71 \pm 6 \%$. Cavity outlines and graphs showing changes in cavity area, estimated volume, perimeter, shape index, and long axis from a representative subject are shown in Fig. I and 2. A similar pattern was seen in all members of the group. End-diastolic shape index was $85 \pm 4 \%$ dropping to $65 \pm 3 \%$, a mean reduction during systole of $20 \pm 3 \%$. The systolic reduction in cavity area was thus mediated

TABLE Summary of changes in ventricular shape and ejection fraction (mean values $\pm I$ standard deviation)

\begin{tabular}{|c|c|c|c|c|c|}
\hline Patients & $\begin{array}{l}\text { Ejection } \\
\text { fraction } \\
(\%)\end{array}$ & $\begin{array}{l}\text { End-diastolic } \\
\text { shape index } \\
(\%)\end{array}$ & $\begin{array}{l}\text { End-systolic } \\
\text { shape index } \\
(\%)\end{array}$ & $\begin{array}{l}\text { Shape } \\
\text { change } \\
(\%)\end{array}$ & $n$ \\
\hline $\begin{array}{l}\text { Normal subjects } \\
\text { Mitral regurgitation } \\
\text { Congestive cardiomyopathy } \\
\text { Anterior or inferior hypokinesia } \\
\text { Left ventricular aneurysm }\end{array}$ & $\begin{array}{l}71 \pm 6.4 \\
60 \pm 20 \\
30 \pm 9.6 \\
64 \pm 10 \\
26 \pm 6\end{array}$ & $\begin{array}{l}85 \pm 4 \\
85 \pm 3 \\
85 \pm 3 \\
81 \pm 5 \\
84 \pm 9\end{array}$ & $\begin{array}{l}65 \pm 3 \\
72 \pm 12 \\
84 \pm 3 \\
73 \pm 7 \\
78 \pm 7\end{array}$ & $\begin{array}{l}20 \pm 3 \\
12 \pm 11 \\
1 \pm 2 \\
8 \pm 5 \\
7 \pm 4\end{array}$ & $\begin{array}{r}10 \\
18 \\
6 \\
10 \\
6\end{array}$ \\
\hline
\end{tabular}



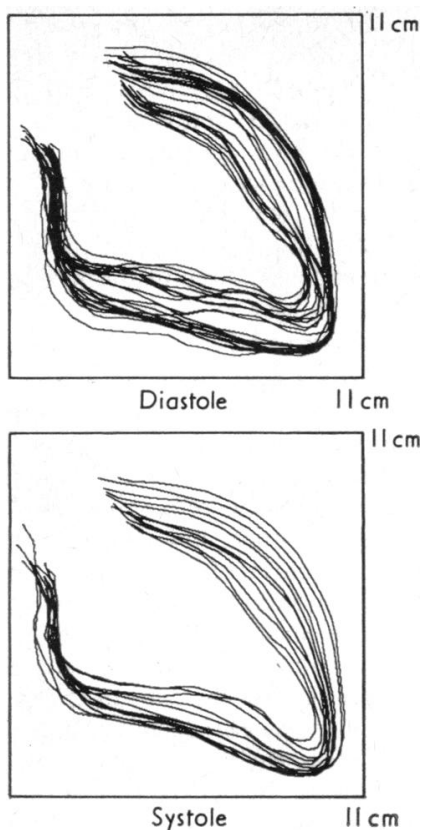

FIG. I Left ventricular outlines in systole (lower frames) and diastole (upper frames) from normal subject.
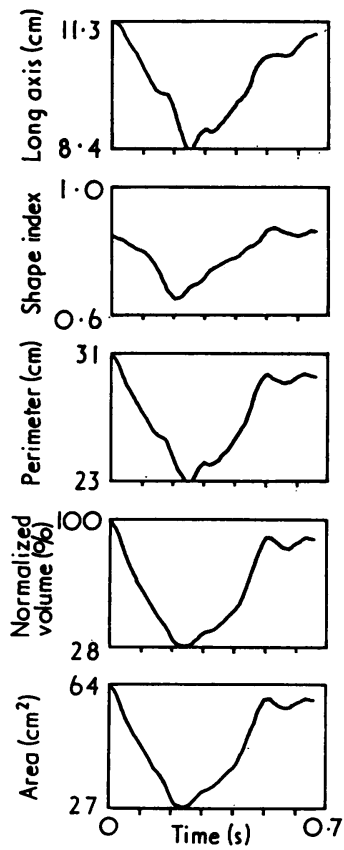

FIG. 2 Changes in cavity area, estimated volume, perimeter, shape index, and long axis, during cardiac cycle illustrated in Fig. $I$. by a change in shape towards a less circular configuration as well as by a reduction in perimeter, both processes occurring concomitantly. During diastole the mechanism of change in cavity area was the reverse of that in systole. Throughout the cardiac cycle rates of wall movement were similar in all regions of the ventricle, as reflected by the uniform spacing of the cavity outlines until the onset of diastasis.

\section{Non-rheumatic mitral regurgitation}

This group included patients with ejection fraction between $20 \%$ and $80 \%$ with a mean value of $60 \pm$ $20 \%$. End-diastolic shape index was identical with that in the normal group, though the mean fall during systole was significantly less ( $12 \pm 11 \%$, $\mathrm{P}<0.0 \mathrm{r})$. In the group as a whole there was very significant correlation between ejection fraction and the change in shape index occurring during systole (Fig. 3). In those patients in whom ejection fraction was greater than $70 \%$ wall movement showed a characteristic and consistent pattern, an example being shown in Fig. 4 and 5. During systole it was concentric and uniform in all parts of the cavity, but in diastole there was an initial rapid movement in the region opposite to the mitral valve followed by downward movement of the apex and finally upward movement of the base. This resulted in an early increase in shape index before significant prolongation of long axis had occurred (Fig. 5).

\section{Congestive cardiomyopathy}

The mean ejection fraction in this group of patients

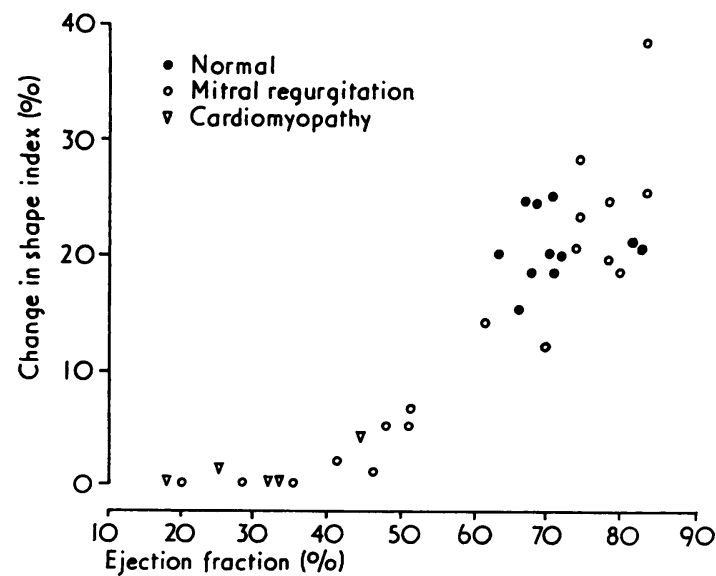

FIG. 3 Relation between ejection fraction and shape index changes in normal subjects and patients with non-rheumatic mitral regurgitation or congestive cardiomyopathy. 

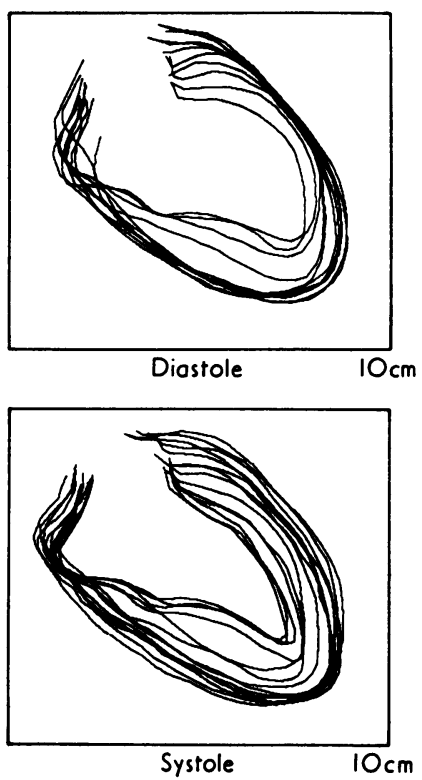

FIG. 4 Cavity outlines from a patient with mitral regurgitation due to ruptured chordae tendineae. Wall movement during systole is concentric, but during diastole outward wall movement occurs initially in a region opposite to mitral valve followed by downward movement of the apex and upward movement of aortic root.
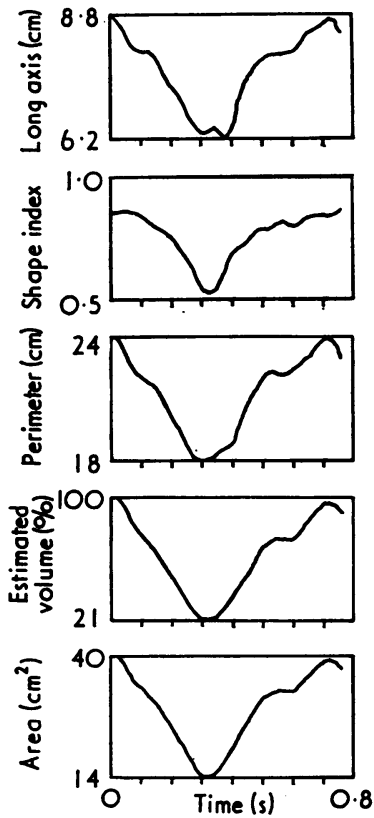

FIG. 5 Changes in cavity area, estimated volume, perimeter, shape index, and long axis, from cardiac cycle illustrated in Fig. 4. was $30 \pm 10 \%$. End-diastolic shape index was normal $(85 \pm 3 \%)$, but there was no significant reduction during systole. Since the cavity was dilated and the stroke volume small overall amplitude of wall movement during the cardiac cycle was small in this group of patients.

\section{Ischaemic heart disease}

In Io patients with localized hypokinesia involving either the anterior or the inferior wall the mean ejection fraction was $64 \pm 10 \%$ and was thus not significantly different from normal. Though enddiastolic shape index was also normal the reduction during systole was significantly less than normal $(8 \pm 5 \%, \mathrm{P}<0.00 \mathrm{I})$. This difference was reflected in the failure of the cavity to become elongated at endsystole, but instead it maintained its diastolic configuration throughout the cardiac cycle (Fig. 6). In six patients with ventricular aneurysm mean ejection fraction was $26 \pm 6 \%$. In spite of this the reduction in shape index during systole was $7 \pm 4 \%$, significantly greater than in patients with congestive cardiomyopathy $(\mathbf{P}<0.00 \mathrm{I})$.

\section{Relation between posteroanterior and lateral projections}

In 15 patients posteroanterior and lateral projections
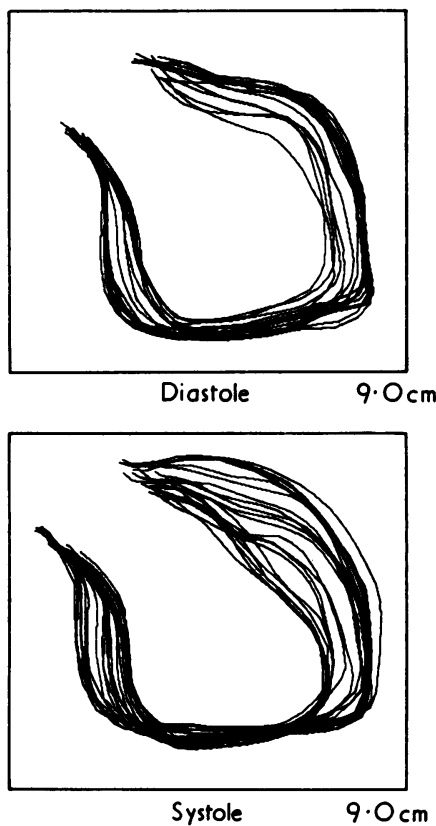

FIG. 6 Cavity outlines from patient with ischaemic heart disease. Though ejection fraction is $63 \%$ the reduction in shape index during systole is only $8 \%$. 
were available. The relation between estimates of ejection fraction derived from the two is given by

(Ejection fraction $)_{\mathrm{PA}}$

$$
\begin{aligned}
& =0.84 \text { (Ejection fraction) })_{\mathrm{LAT}}+\mathrm{II} \% \\
& r=0.92 \text {, standard error of estimate }=7 \%,
\end{aligned}
$$

and between the two estimates of shape index change by

(Shape change) $)_{P A}=0.82$ (Shape change) $)_{\mathrm{LAT}}+2 \%$ $r=0.90$, Standard error of estimate $=3 \%$.

Agreement between estimates of the change in shape index derived from the two planes is thus almost identical to that between estimates of ejection fraction.

\section{Discussion}

The function of the heart as a pump depends on the accumulation 'of blood in the ventricular cavity during diastole and its ejection with the succeeding systole. These volume changes can be outlined by angiocardiography, and the properties of the resulting images described (Dodge et al., 1966; Sandler and Alderman, 1974). A fundamental property of any solid is the relation between its volume and the nature and extent of the boundary that surrounds it, with an analogous relation in two dimensions between area and perimeter. In the present study this has been used as the basis for a definition of shape and used to investigate changes in cavity configuration during the cardiac cycle. The anatomical basis of these changes is complex but, as has previously been pointed out, this basis need not be specified.

It is not possible to obtain a comprehensive picture of the left ventricular cavity by standard angiocardiographic methods (Johnson et al., 1974). The outline of the projection obtained by single plane angiocardiography cannot be assumed to be anatomically continuous but may be the resultant of several overlapping boundaries if concave as well as convex regions are present. The same potential limitation applies to biplane angiocardiograms, so that in the present study these have been used merely to provide two arbitrary projections whose properties can be compared to give some idea of uniformity of behaviour in different regions of the cavity. The similarity of ejection fraction and shape index changes in the two projections provides evidence that left ventricular contraction is a uniform process, and suggests that it is possible to extrapolate from a single projection to the properties of the cavity as a whole.

Angiocardiography has well recognized limitations in defining the endocardial surface of the left ventricular wall. It has previously been shown that the outer border of the opacified left ventricular cavity may be separated from radio-opaque markers on the endocardium itself, particularly at endsystole, probably owing to endothelial infolding (Mitchell, Wildenthal, and Mullins, 1969). Similarly, cavity obliteration may occur at the apex, while at end-systole the margin of the cavity may be formed by the papillary muscles rather than the free wall of the ventricle. These considerations do not affect the present study in which the left ventricular cavity was defined as the region accessible to angiographic dye, though they do stress difficulties inherent in studying myocardial mechanics by angiocardiographic methods.

The use of a definition of shape based on the properties of the cavity as a whole has certain advantages. It is not dependent on the position of the zero reference used for defining cavity outlines, and so is insensitive to overall movement of the ventricle in space. It does not require the definition of any fixed point on the perimeter such as the 'apex', which may be difficult in the presence of abnormal wall movement or aneurysm. It assumes no idealized cavity geometry and, being dimensionless, is unaffected by cavity size. Finally, it is not invalidated by local abnormalities of wall movement.

In contrast, techniques based on the superimposition of an arbitrary grid depend on identification of the apex or are sensitive to overall movement of the heart in space. In general, these methods assess wall movement only at the point where the axis and cavity outline intersect one another. Localized abnormalities of wall movement might thus be missed altogether or may cause relative movement of the axes and the myocardium with the effect that different regions of the ventricle are studied at different times in the cardiac cycle. Disadvantages from the use of the present method may arise from errors in defining the left ventricular margin on either side of the true perimeter and also, with increasing resolution of angiocardiographic equipment, from digitizing the trabecular fine-structure of the wall. Both of these effects will cause an increase in perimeter without a proportional increase in cavity area. Both could have been minimized by an alternative definition of shape such as one in which cavity area is divided by that of the circle that just encloses it. However, comparison of these two methods showed that in representative patients there was no significant difference between them in assessing the extent or timing of shape changes.

Left ventricular shape changes during systole were described by William Harvey (I628), who noted that at this time the heart became 'narrower, relatively longer and more drawn together'. Similar 
observations have been made by subsequent observers in animals (Hawthorne, I96I; Ross et al., 1967; Bove, 1971; Streeter and Hanna, 1973) and in man (Hood and Rolett, 1969; McDonald, 1970; Vokonas et al., 1973; Gould et al., 1974). In normal subjects this process occurred concomitantly with changes in long axis, cavity area, and perimeter. A reduction in shape index occurred only in ventricular systole and so appeared to be the result of an active and irreversible process associated with the development of stress in the wall, with subsequent expenditure of work on the circulation. This shape change has a clear functional significance, since in its absence similar reduction in perimeter would lead to a smaller change in area. When extrapolated to ventricular volume this shows that, in the absence of any shape change, stroke volume would be reduced by approximately one third.

Ventricular filling was accompanied by an equal and opposite change in shape to that occurring during systole, which, in normal subjects, also proceeded concomitantly with those in area, perimeter, and long axis, during early diastole. In all the patients studied shape index either remained unchanged or increased in diastole, suggesting that a reduction in shape index does not occur when work is done on the ventricle by the circulation. Diastolic shape changes have functional significance in that the inflow of blood during the rapid filling phase is partly accommodated by the cavity becoming more spherical, so that the rate of myocardial distension is correspondingly reduced. It follows, therefore, that at end-systole the configuration of the cavity is one which aids filling but which can be brought about only by an active process in the ventricular wall rather than by work done on it by the circulation. The process of filling during early diastole is thus likely to be profoundly different in the beating heart from that in an arrested specimen.

Though patients with reduced ejection fraction had a normal end-diastolic shape index little or no reduction occurred with systole, so that the cavity projection remained more nearly circular throughout the cardiac cycle. Reduced shape index changes also occurred in patients with anterior or inferior hypokinesia in spite of ejection fraction being within the normal range. In these patients, therefore, stroke volume can have been maintained only by proportionately more muscle shortening than would have been the case if the normal shape index change had occurred, providing a mechanism whereby localized abnormalities of contraction induced by ischaemia can lead to generalized impairment of ejection. In addition, the pattern of filling might also be disturbed with a larger component of inflow being accommodated by stretching of the myocardium rather than by the ventricle becoming more spherical. It follows that diastolic ventricular compliance measured in terms of pressure volume relations may vary independently of myocardial stiffness if shape changes occur, so that it is not possible to predict one of these variables from measurement of the other.

In patients with mitral regurgitation due to ruptured chordae tendineae in whom left ventricular function is normal, stroke volume may be very large. In these patients the characteristics of ejection were similar to those of normal subjects, though in some cases the changes were more extensive. In diastole, however, there were significant differences. In the early phase of ventricular filling there was a striking increase in transverse diameter resulting in the cavity becoming more circular. This was followed by downward movement of the apex and upward movement of the base at a time when shape index changes were virtually complete (Fig. 4 and 5). These changes presumably represent adaptation to the very rapid filling that occurs in the presence of severe mitral regurgitation and indicate that, in these circumstances, filling can be a strikingly non-uniform process. This may represent the effect of failure to attain equilibrium conditions during early diastole, of regional variation in distensibility at this time in the cardiac cycle, or of constraint by the pericardium. It is apparent, however, that a full description of the diastolic properties of the left ventricle must include information about their spatial distribution as well as their variation with time as filling proceeds.

In patients in whom ejection fraction was low shape index changes were small or undetectable. This could not be related to end-diastolic shape itself, which was not significantly different from normal. A second possibility was that, since the overall amplitude of wall movement was reduced, there was a corresponding reduction in the potential for shape changes. This effect is clearly an important one, but had it been the only one a progressive reduction in shape index with reducing ejection fraction might have been expected rather than the observed response, in which shape index changes were linearly related to ejection fraction for values above $50 \%$ and undetectable below (Fig. 3). Probably, therefore, the absence of shape change reflects the additional effect of distortion or loss of normal ventricular architecture when the cavity is dilated.

In contrast to patients with localized hypokinesia those with a ventricular aneurysm showed well marked shape changes in spite of ejection fraction being very low. This probably reflected the different type of disturbance of ventricular function in the two groups of patients. In those with ventricular 
aneurysm, systolic volume changes in the normally functioning region are in the opposite direction from those in the part behaving aneurysmally, with the result that ejection fraction is low while both regions become distorted during systole so that the corresponding shape changes are additive. This effect might provide a means whereby the disturbance to left ventricular function caused by aneurysm might be quantified without the necessity for defining the boundary between normal and abnormal regions.

The present observations also stress differences in the relation between cavity configuration, pressure, and wall stress in systole and diastole. In diastole, when the ventricle behaves passively, pressure and wall stress increase as a result of work done on the heart by the circulation, and under these conditions shape index remains unchanged or increases. By contrast, during systole increased wall stress is associated with a reduction in shape index. This difference is not reflected in current methods for estimating wall stress based on Laplace's law, which predicts that the cavity should always become more spherical with increasing wall tension. This major discrepancy between theory and observation shows that a more complex model is required for deriving systolic wall stress. This may have to include information about local variation in structure, fibre orientation, and time-dependence of the active elements during the contractile process. More detailed observations of changes in cavity shape may be helpful in evaluating such models and thus of determining the structural basis of shape changes in normal and abnormal hearts.

\section{References}

Bove, A. A. (1971). Radiographic evaluation of dynamic geometry of the left ventricle. Fournal of Applied Physiology, 31, 227.

Dodge, H. T., Sandler, H., Baxley, W. A., and Hawley, R. R.
(1966). Usefulness and limitations of radiographic methods for determining left ventricular volume. American fournal of Cardiology, 18, I0.

Gibson, D. G., and Brown, D. J. (1975). Measurement of peak rates of left ventricular wall movement in man. British Heart fournal, 37, 677.

Gould, K. L., Lipscomb, K., Hamilton, G. W., and Kennedy, J. W. (1974). Relation of left ventricular shape, function and wall stress in man. American fournal of Cardiology, 34, 627.

Harvey, W. (1628). On the motion of the heart and blood. De motu cordis et sanguinis. Translated by Willis, R., p. 24 . Dent, Everyman, London, 1907.

Hawthorne, E. W. (I96I). Instantaneous dimensional changes of the left ventricle in dogs. Circulation Research, 9, 110.

Holt, M., and Marjoram, D. T. E. (1973). Measurement of shape in geography. In Mathematics in a Changing World, p. 236. Heinemann, London.

Hood, W. P., and Rolett, E. L. (1969). Patterns of contraction in the human left ventricle (abstract). Circulation, 40, Suppl. III, IO9.

Johnson, S. A., Robb, R. A., Greenleaf, J. F., Ritman, E. L., Lee, S. L., Herman, G. T., Sturm, R. E., and Wood, E. H. (1974). The problem of accurate measurement of left ventricular shape and dimension from multiplane roentgenographic data. European fournal of Cardiology, r, 241 .

McDonald, I. G. (1970). The shape and movements of the human left ventricle during systole. American fournal of Cardiology, 26, 221.

Mitchell, J. H., Wildenthal, K., and Mullins, C. B. (1969). Geometrical studies of the left ventricle utilizing biplane cinefluorography. Federation Proceedings, 28, 1334 .

Ross, J., Jr., Sonnenblick, E. H., Covell, J. W., Kaiser, G. A., and Spiro, D. (1967). The architecture of the heart in systole and diastole: technique of rapid fixation and analysis of left ventricular geometry. Circulation Research, 21, 409.

Sandler, H., and Alderman, E. (1974). Determination of left ventricular size and shape. Circulation Research, 34, I.

Streeter, D. D., and Hanna, W. T. (1973). Engineering mechanics for successive states in canine left ventricular myocardium. Circulation Research, 33, 639.

Vokonas, P. S., Gorlin, R., Cohn, P. F., Herman, M. V., and Sonnenblick, E. H. (1973). Dynamic geometry of the left ventricle in mitral regurgitation. Circulation, 48, 786.

Requests for reprints to Dr. Derek Gibson, Cardiac Department, Brompton Hospital, Fulham Road, London $\mathrm{SW}_{3}$ 6HP. 